\title{
CONSIDERACIONES CLAVE PARA EL DEBATE SOBRE LA MINERÍA METÁLICA EN EL SALVADOR
}

\author{
Andrés McKinley
}

Recibido: 24/04/2016 Aceptado: 06/09/2016

\begin{abstract}
Resumen
El presente artículo plantea la larga y dura batalla, por más de una década contra la minería metálica en El Salvador y analiza los factores clave que influyen en el debate en torno a este tema. La discusión se centra en los altos niveles de deterioro ambiental que actualmente padece este país y que lo dejan en una situación de especial vulnerabilidad frente a la amenaza de la minería industrial. Describe en detalle la amenaza de la minería metálica al medio ambiente a nivel mundial, sobretodo a los recursos hídricos del planeta; explica la amenaza específica de la minería metálica al desarrollo sostenible en El Salvador, donde la mayoría de los depósitos de oro y plata están situados en la cuenca del estratégico río Lempa; plantea el balance desfavorable entre los costos y los beneficios de la minería metálica para países como El Salvador, y analiza el amplio rechazo de la ciudadanía e instituciones clave a dicha industria. El artículo se cierra con una discusión y recomendaciones sobre los desafíos de la buena gobernanza, la necesidad de políticas públicas claras y efectivas y el desafío a largo plazo de la construcción de alternativas al modelo extractivo para el desarrollo de los países.
\end{abstract}

Palabras clave: minería metálica; estrés hídrico; contaminación; cianuro; El Salvador.

\begin{abstract}
The current article presents El Salvador's decade-long battle against metallic mining and analyzes the key factors which influence the debate around this theme. The discussion focuses on the high levels of environmental deterioration that render the country uniquely vulnerable to the impacts of industrial mining, especially on scarce and threatened water resources. It describes in detail the threat of metallic mining to the global environment, especially water; explains the specific threat of metallic mining to sustainable development in El Salvador, where the majority of gold and silver deposits are located in the watershed of the highly strategic Lempa River; points to the unfavorable balance between the costs and benefits of metallic mining to countries like El Salvador and analyzes the almost unanimous rejection of this industry by its citizens and key institutions. The article closes with a discussion and recommendations on the challenges of good governance, the need for clear and effective public policies, programs and practices and the long-term challenge of building alternatives to the current extractive model for development.
\end{abstract}

Key words: metallic mining; water stress; contamination; cyanide; El Salvador. 


\section{Introducción}

En la última década, la inversión de la industria minera ha crecido de manera dramática alrededor del mundo, sobretodo en países en vías de desarrollo. Este fenómeno se debe a una variedad de factores, pero responde principalmente al aumento de la demanda de minerales de naciones como China e India, al incremento en los precios de los metales en el mercado mundial, a la especulación en materias primas a partir del colapso del mercado financiero en el 2008 y a la existencia de leyes en países en vías de desarrollo diseñadas para atraer la inversión extranjera. Entre el 2005 y el 2010, el sector minero de China creció más de 30 por ciento con inversiones en África y América Latina (SEPA MÁS, 2015), mientras que las empresas canadienses, australianas, estadounidenses y sudafricanas fortalecieron su inversión en la minería metálica alrededor del mundo.

En este contexto, la explotación de oro y plata ha sido problemática debido a los enormes daños en los ecosistemas, los altos costos que ha dejado para las comunidades cercanas (especialmente de pueblos indígenas) y su impacto negativo en las formas tradicionales de vida de poblaciones locales a lo largo de América, África, Asia y otras partes del mundo.

Más de 70 por ciento de los proyectos mineros de oro y plata está ubicado en países en vías de desarrollo, donde las poblaciones cercanas han sido víctimas del acaparamiento de sus tierras, de la destrucción de sus bosques y montañas (considerados sagrados en muchas culturas autóctonas, como los mayas de Guatemala), del daño, a veces irreversible, de ecosistemas y de la contaminación de sus fuentes de agua dulce (McKinley, 2015a). En la mayoría de estos países, la incursión minera ha generado mayores niveles de pobreza y corrupción, amenazado procesos incipientes de democratización, generado conflicto, afectado el tejido social de comunidades cercanas y ha violado los derechos humanos más fundamentales de los sectores pobres, especialmente de los pueblos indígenas (McKinley, 2015a).

El Salvador es uno de los países más amenazados por la nueva ola de minería metálica, donde empresas mineras canadienses, estadounidenses y australianas han querido explotar depósitos de oro y plata en un cinturón que atraviesa el norte del país, pasando por zonas estratégicas de recarga hídrica y ubicadas, en su mayoría, en la cuenca del río Lempa, la fuente más importante de agua para usos domésticos, industriales, agrícolas y energéticos (McKinley, 2015b).

Comunidades amenazadas, ONG ambientalistas, iglesias y movimientos sociales, como la Mesa Nacional Frente la Minería Metálica, llevan más de una década luchando contra la incursión de empresas mineras trasnacionales en el país. La Conferencia Episcopal de la Iglesia católica, en el 2007, se sumó a este esfuerzo con su pronunciamiento "Cuidemos la Casa de Todos". A este colectivo socioambiental pertenecen la Universidad Centroamericana, José Simeón Canas (UCA), junto con 
Caritas, organizaciones ambientalistas (CESTA), consumidores (CDC), organizaciones de derechos humanos (Tutela Legal) y de desarrollo comunitario (COFOA), con lo cual incorporaron a nuevos sectores de inspiración cristiana en la lucha.

Desde el 2009, tres gobiernos sucesivos de El Salvador, presionados por la ciudadanía, han mantenido una moratoria de facto de la minería metálica en el país, al negar permisos nuevos de exploración y rechazar solicitudes para concesiones de explotación. Esto llevó a la empresa canadiense, Pacific Rim, la cual operaba un proyecto de exploración de oro y plata en el municipio de San Isidro, departamento de Cabañas, a demandar al Estado salvadoreño ante el Centro Internacional para el Arreglo de Diferencias Relativas a Inversiones (CIADI) del Banco Mundial en Washington, D.C. por un monto de US\$77 millones (que subió después a US\$250 millones) (Marroquin, 2016).

Pacific Rim argumentó que su permiso de exploración automáticamente le daba derecho a una licencia de explotación (un reclamo falso, según la actual Ley de Minería de El Salvador), que la empresa había cumplido con todos los requisitos legales para una licencia de explotación de oro y plata (también falso) y que el Gobierno había sugerido que su solicitud sería aprobada. En su defensa, el Estado de El Salvador ha argumentado que Pacific Rim nunca cumplió con los requistos establecidos en el actual Ley de minería del país. Además, la empresa nunca presentó un estudio de impacto ambiental aceptable, no finalizó su estudio técnico de factibilidad y ni comprobó el control legal del territorio a minar. Sin otros proyectos mineros en el mundo y sin fuentes de ingreso, Pacific Rim empezó a experimentar graves problemas financieros a partir de 2013 y terminó vendiéndose por US\$12 millones a la empresa australiana OceanaGold, que actualmente lleva el caso en CIADI.

Ha sido un proceso de siete años, considerado uno de los más lentos en los 50 años de existencia de CIADI. Hasta el momento, el Estado salvadoreño ha gastado más de US\$13 millones en su propia defensa, recursos que pudo haber invertido en salud, educación, seguridad pública, producción alimentaria, promoción de equidad de la mujer, entre otras prioridades.

Ante una injusticia tan evidente y ante el precedente tan importante del caso, activistas, centros de investigación, movimientos ambientalistas, iglesias y organizaciones de derechos humanos en EE. UU., Canadá, Australia, Filipinas y muchos otros países han seguido el proceso con gran interés. Por ejemplo, hace unos años se formó la agrupación "Aliados Internacionales Contra la Minería Metálica en El Salvador", un conjunto de organizaciones estadounidenses, canadienses y australianas comprometidas con la lucha de las comunidades y del pueblo salvadoreño en contra de la minería metálica. Entre los Aliados se encuetran organizaciones muy conocidas y de mucha trayectoria, como el Institute for Policy Studies y el Center for International Environmental Law (CIEL), en Washington, D.C.; Mining Watch Canada, OXFAM, US Sister Cities, CISPES, Washington Ethical Society, Council of Canadians, Maritime Union of Australia, International Trade Union Congress, el AFL-CIO, el Sierra Club, Friends of 
the Earth, Chicago Religiouos Leadership Network, Maryknoll Sisters, United Church of Canada, Casa Maryland, Latin American Working group (LAWG), Sisters of Mercy y Common Frontiers Canada.

El 14 de octubre del 2016, el CIADI finalmente anunció su laudo en el caso de la demanda de Pacific Rim/OceanaGold contra el Estado salvadoreño, el cual falló a favor de este país, al determinar que el caso de demanda no tenía mérito, por lo que se exigió un reembolso de gastos legales al Estado por US\$8 millones. Sin embargo, OceanGold todavía insiste en su derecho e intención de minar oro y plata en El Salvador y rechaza la obligación de realizar dicho pago. De este modo, el fallo de CIADI representa una victoria histórica para el país frente a una transnacional que se niega a escuchar la voz de las poblaciones más amenazadas por la minería, y también genera una oportunidad para lograr la aprobación de leyes que prohiban la minería metálica y regulen la minería no metálica en el país.

La amplia y prolongada lucha contra la minería metálica en El Salvador, tanto a nivel nacional como internacional, con el caso de Pacific Rim/OceanaGold como punto de lanza, ha generado una expectativa enorme entre los países en vías de desarrollo, en un mundo que sufre de un desbalance de poder entre gobiernos soberanos y empresas trasnacionales. Junto con Costa Rica, país que estableció una prohibición total de la minería metálica a cielo abierto y el uso de químicos tóxicos (veáse el artículo de Josué Arévalo en este mismo volumen), El Salvador es de los primeros países en el mundo en analizar cuidadosamente los costos y beneficios de esta industriala y ejercer su derecho a decir "no" a su instalación en el territorio nacional.

Este artículo, a partir de un enfoque de derecho y de sustentabilidad, tratará de analizar y explicar dicho fenómeno, en respuesta a varias preguntas centrales: ¿Cuáles son las consideraciones claves, que explican la postura de rechazo de la ciudadanía y del Estado de El Salvador frente la ofensiva de corporaciones transnacionales interesadas en explotar los minerales metálicos? ¿Cuáles son los factores de fondo que explican el surgimiento y fortalecimiento de una lucha social persistente en El Salvador e internacionalmente, capaz de cerrar la puerta a la agresión de empresas transnacionales mineras por más de una década? ¿Por qué es tan injusta la intención de empresas mineras transnacionales, como Pacific Rim y OceanaGold de forzar al país a abrirse a la explotación minera a través de demandas en cortes internacionales, como CIADI?

\section{El tema del medio ambiente-asunto de vida o muerte en El Salvador:}

La discusión sobre la minería metálica en países como El Salvador, en general, se enfoca en el tema del medio ambiente; pues los recursos naturales del planeta, especialmente el agua, están en peligro por la mala gestión, el consumo exagerado, la contaminación y el cambio climático (McKinley, 2015c). En el caso salvadoreño, la situación se ha convertido en un tema de vida o muerte. 
Aunque los medios de comunicación del país más poderosos no lo reflejan así, para este país, el cuido del medio ambiente es prioritario y determinante para la viabilidad socio-económica y para la vida misma. El futuro de El Salvador, como nación, depende, de manera especial, del estado de sus recursos naturales, sobre todo del agua. Es un tema que definirá si las generaciones del futuro se quedan, producen y desarrollan este pequeño espacio geográfico en Centroamérica, o si la opción de vida más atractiva para la juventud seguirá siendo la migración hacia los Estados Unidos.

Según las Naciones Unidas, El Salvador posee el mayor nivel de deterioro ambiental en el hemisferio occidental, después de Haití (PNUMA, 2010). La Organización de las Naciones Unidas para la Alimentación y la Agricultura (FAO, por sus siglas en inglés) ubica a El Salvador entre los países más deforestados de Centroamérica con solo un 3 por ciento de bosque natural y un 13 por ciento de territorio cubierto de bosque (comparado con Costa Rica que tiene más de 50 por ciento de cobertura forestal). Aunado a esto, ha sido calificado por las Naciones Unidas, la CEPAL y el Tribunal Latinoamericano del Agua como la nación de menor disponibilidad de agua por habitante por año en todo el continente americano, después de Haití (Rivas, 2010). La Alianza Mundial para el Agua (GWP, por sus siglas en inglés) indica que El Salvador se acerca a una situación de estrés hídrico (cuando la disponibilidad de agua por persona por año llega a $1700 \mathrm{~m}^{3}$ ) y el mismo gobierno, en abril del 2016, declaró estado de emergencia por la falta del este líquido en comunidades tanto rurales como urbanas.

Para volver más complejo este escenario, el Ministerio de Medio Ambiente y Recursos Naturales (MARN) de El Salvador ha informado que 90 por ciento del agua superficial disponible en el país está seriamente contaminada y que solo el 11 por ciento de las aguas superficiales son aptas para ser potabilizadas por medios convencionales (Mendoza y Sosa, 2016).

Por todo lo anterior, las Naciones Unidas ubica a El Salvador entre los países más vulnerables del mundo frente al cambio climatico y los desastres naturales (OXFAM International, 2014). Así, en estas condiciones de extrema vulnerabilidad, permitir la incursión de industrias que impactan de manera dramática al medio ambiente, especialmente a los recursos hídricos, sería, en la mente de muchos, un suicidio colectivo.

\section{La amenaza de la minería metálica al medio ambiente}

El planeta Tierra ya muestra las heridas y cicatrices de miles de años de minería metálica. Las reservas minerales que quedan en su subsuelo tienden ser de baja concentración y de poca calidad. En el caso de los depósitos de oro, es común encontrar la explotación de concentraciones de menos de 10 gramos de oro por tonelada de roca, por lo cual se requiere procesar más de 20 toneladas de material para lograr una 
sola onza de oro, cantidad requerida para elaborar un anillo. Algunos analistas se refieren a estos depósitos con términos como "oro microscópico" u "oro invisible".

La explotación de depósitos minerales de baja calidad requiere de tecnologías extremadamente dañinas para el medio ambiente. A pesar de los intentos de la industria minera por ganar mentes y corazones con la promesa de que existen nuevas tecnologías armoniosas con la naturaleza (utilizando términos como "minería verde", "minería moderna" o "minería responsable"), no hay nada nuevo que permita la explotación de minerales de baja concentración sin dañar al medio ambiente. La minería metálica impacta los recursos hídricos por el consumo exorbitante de agua, por la destrucción de bosques y suelo, la cual disminuye la capacidad de recarga hídrica, y por la contaminación de ríos, lagos, acuíferos y océanos (McKinley, 2015c).

\section{El consumo exorbitante del recurso hídrico}

El agua es esencial para todas las fases de la minería metálica moderna, desde la exploración hasta la extracción y procesamiento de minerales. Se puede decir que el agua es la "sangre" o "fuente de vida" de esta minería y que compite con los seres humanos por esta "fuente de vida". Las minas de oro y plata de Centroamérica, según datos de las mismas empresas, consumen más de un millón de litros de agua diarios (McKinley, 2015a).

En la etapa de exploración, las empresas mineras hacen cientos de perforaciones profundas en la tierra (hasta de 400 metros) para comprobar la presencia y el grado de concentración del oro. En este proceso es frecuente que los mantos acuíferos y las fuentes de agua de las comunidades cercanas se afecten, como sucedió entre el 2007 y el 2009 en el proyecto minero, El Dorado, en Cabañas, El Salvador. En este caso, la empresa minera canadiense, dueña del proyecto, terminó secando más de veinte nacimientos históricos de agua en las comunidades locales (Broad y Cavanagh, 2011).

Existen casos similares a través de todo Centroamérica, por ejemplo, en sus operaciones diarias, se calcula que la mina Marlin (ya en proceso de cierre), en el departamento de San Marcos del vecino país de Guatemala, utilizaba unos 250,000 litros de agua por hora, es decir, unos 6 millones de litros diarios, cantidad mayor a la que usa una familia campesina en 30 años. Comunidades que viven cerca de la mina han reportado más de 40 pozos comunales secos en los primeros diez años de vida de este proyecto. Por su parte, una mina de níquel a la orilla del Lago Izabal del mismo país, conocida como el proyecto Fénix y ya en manos de una empresa rusa, utiliza diariamente 13 veces la cantidad de agua requerida para la ciudad más cercana de El Estor; y en Honduras, pobladores de Valle de Siria, una zona históricamente ganadera y de producción de granos básicos, reportan que la mina San Martín, en 9 años de operaciones, secó 19 de los 23 ríos originales de la zona (véase artículo de Nick Middeldorp en este mismo número). 
En la mayoría de casos, hay poco monitoreo o control gubernamental sobre el uso de agua por las empresas mineras y raramente se paga por el acceso, aun cuando reduce la cantidad de agua disponible para otros usos, como el doméstico, agrícula, industrial y energético.

\section{Disminución en la capacidad de recarga hídrica}

La minería a cielo abierto destruye los bosques, daña los suelos de uso agrícola y disminuye la capacidad de la tierra para absorber las aguas llovidas, lo cual aumenta el riesgo de erosión e impacta la capacidad de recarga de acuíferos, ríos y lagos. Este peligro es especialmente latente en El Salvador, donde siglos de mala práctica en la agricultura han destruido los suelos y los bosques del país.

El Salvador es un país que recibe abundante lluvia cada año, incluso en los últimos años, con el fenómeno del "Niño", pero la destrucción de bosques y suelo a través de los siglos de mal manejo ha generado una situación en la cual la lluvia que cae sobre el país, en lugar de ser absorbida por el suelo para la recarga de acuíferos, ríos y lagos, se lava hacia el mar.

Contaminación de ríos, lagos, acuíferos y océanos

La minería metálica ocupa una variedad de químicos y otras sustancias altamente tóxicas en las etapas de exploración, extracción y procesamiento, incluyendo el mercurio, cianuro de sodio, ácido sulfúrico y ácido hidroclórico. Utiliza explosivos durante la etapa de extracción que dejan residuos solubles de nitrato y amonio y requiere de enormes cantidades de combustible, aceite y anticongelante que están embodegados en el sitio de la mina. Todos estos materiales generan el riesgo de fuga y de filtración en el curso de su uso normal y son una amenaza grave para el agua y a la vida acuática.

Varias de las empresas mineras más grandes del mundo han sido culpables de verter desechos de la minería directamente a los ríos, lagos y océanos, contaminando ecosistemas, obstaculizando ríos con sedimentos e interrumpiendo la hidrología de cuencas completas (Sumi y Gestring, 2013). Empresas mineras en Chile, país ejemplificado en la propaganda pro-minera en Centroamérica, ya no tienen donde tirar sus desechos y han planteado como solución la canalización de miles de toneladas de residuos a través de ductos gigantescos para depositarlos en una especie de valle en el fondo del mar (Ortúzar y Rodríguez, 2015).

El peligro del cianuro

El cianuro de sodio es un químico altamente tóxico, aplicado en cantidades abundantes (miles de galones diarios) en la minería de oro y plata. Puede ser fatal para 
los seres humanos en cantidades del tamaño de un grano de arroz y es letal para la vida acuática en concentraciones bajas. Por eso, ha sido prohibido en varias regiones del mundo, incluyendo República Checa, Grecia, Turquía, Alemania, Hungría, Costa Rica, algunas provincias de Argentina y Ecuador y ciertas regiones de Estados Unidos (Montana y Colorado). No obstante, se usa con frecuencia, y sin controles, en la mayoría de minas de oro y plata de los países en vías de desarrollo, como los de Centroamérica, con abundantes casos documentados de fuga lo cual impacta la vida acuática y la disponibilidad de fuentes de agua dulce para los seres humanos en la región. Ejemplos incluyen el río Lara de Honduras (Tribunal Centroamericano del Agua, 2004), el río Tzalá de Guatemala (COPAE, s. f.) y el río Bambana de Nicaragua (Movimiento Mesoamericano Contra el Modelo Extractivo Minero, 2013).

Los diques de cola

Una de las fuentes más problemáticas de contaminación por cianuro y otros tóxicos es la falla de "diques de cola", los grandes depósitos donde las empresas mineras acumulan los desechos que van quedando del proceso minero. La "cola" de mina contiene docenas de sustancias peligrosas que son una amenaza a la vida humana y a los ecosistemas. Desde mediados del siglo XX, se han registrado fallas en más de 200 de ellos (Shahid y Qiren, 2015).

El caso más reciente, y tal vez uno de los más dramáticos, ha sido el rompimiento del dique de cola Fundao en la mina Samarco (de las empresas BHP Biliton Ltd y Vale S.A.) en Brasil, en noviembre de 2015. Este se ha catalogado como el peor desastre causado por el hombre en la historia del país, pues generó una inundación con más de 50 millones de metros cúbicos de lodo y desechos tóxicos de la minería, aplastando comunidades, matando a 19 personas, contaminando el río Doce hasta su desembocadura en el mar Atlántico y destruyendo los medios de vida de miles de familias en la región (La Gaceta, 2015).

\section{El drenaje ácido de mina}

El drenaje ácido, tal vez, representa el peligro más grave de contaminación del agua causada por la minería metálica. Ocurre cuando la roca extraída del subsuelo contiene sulfuros, lo cual es frecuente en la minería de oro y plata, en especial en zonas volcánicas, como El Salvador y los demás países de Centroamérica. Cuando esta roca con sulfuros se quiebra, se muele y se expone al oxígeno del aire y al agua de las lluvias, se genera una reacción química que produce sulfatos. Estos, a su vez, se transforman en ácido sulfúrico, el cual se filtra hacia los ríos, lagos y acuíferos en el área de la mina, liberando otros metales de la roca, como cadmio, magnesio, arsénico, plomo, mercurio y hierro que, también, llegan a las fuentes cercanas de agua dulce, 
generando niveles de toxicidad capaces de matar todas las formas de vida acuática y amenazar la vida humana.

El drenaje ácido es difícil de detectar durante la vida de una mina. Normalmente, aparece hasta después del cierre cuando la empresa minera se ha ido del país, llevando con ella la riqueza natural de las comunidades y de las naciones, dejando los enormes costos relacionados con la destrucción ambiental y la contaminación. Una vez que comienza, este fenómeno es virtualmente irreversible. Por ejemplo, hay minas en España y Francia de la época del Imperio romano que continúan generando drenaje ácido.

El Salvador, con una historia minera de menor relevancia, exibe uno de los casos más dramáticos en Centroamérica de este fenómeno. Está ubicado en el municipio de Santa Rosa de Lima del departamento de La Unión, donde hubo minería de oro de pequeña escala hace décadas y donde cientos de comunidades han perdido, para siempre, su fuente principal de agua: el Río San Sebastián. Es claro que las consideraciones anteriores son importantes a tomar en cuenta en un país que padece ya de crisis ambiental, con una tendencia clara hacía el estrés hídrico.

\section{El riesgo de la minería metálica al desarrollo sostenible}

Para ganar mentes y corazones, las empresas mineras hablan con frecuencia sobre la contribución que hace esta industria al desarrollo sostenible. No obstante, es importante recordar que la minería metálica explota recursos no renovables. Una vez que se extrae el oro u otros minerales del subsuelo y lo llevan hacia afuera, no vuelven nunca a la tierra. Así que no existe nada "sostenible" de esta actividad. De hecho desde hace años se ha venido planteando un cuestionamiento fuerte de la contribución que hace la minería en términos del desarrollo de los paises con altos indices de pobreza, como El Salvador (Sachs y Warner, 1997; Ross, 2001; Power, 2008).

Ross (2001), por ejemplo, concluyó que no solo existe una fuerte correlación entre la minería y los altos índices de pobreza, sino que, además, en países dependientes de la explotación de minerales, la expectativa de vida era más baja, la mortalidad infantil mayor, los niveles de equidad en la distribución de la riqueza se tornaban menores, había mayor fluctuación en las economías y mayores niveles de corrupción, ineficiencia gubernamental, autoritarismo, militarización y violencia. Incluso el mismo Banco Mundial (2002), que ha promovido la minería en países en vías de desarrollo alrededor del mundo, ha tenido que aceptar los resultados de sus propios estudios lo cuales indican que las economías de países dependientes de metales para sus exportaciones tienden a crecer a un ritmo más lento que países sin metales.

La Comisión Mundial del Ambiente y Desarrollo de las Naciones Unidas define el desarrollo sostenible como un proceso para "lograr las necesidades del presente sin comprometer las posibilidades de las generaciones futuras para alcanzar sus propias 
necesidades" (traducción en español del autor) (ONU, s. f.). Como ya se planteó, las "posibilidades de las generaciones futures" de El Salvador dependen de la situación de sus recursos naturales, especialmente del agua.

\section{La importancia del río Lempa}

En El Salvador, el desarrollo sostenible es sinónimo del río Lempa, arteria principal y fuente de vida para gran parte de la población del país. Nace en Guatemala, pasa por Honduras, cruza la zona norte de El Salvador y desemboca en el Océano Pacífico. Su cuenca multinacional posee un área total de 17790 km², de los cuales $10082 \mathrm{~km}^{2}$ corresponden a El Salvador, es decir 47,91 por ciento del territorio nacional. Su rompeaguas en El Salvador se extiende por $360.2 \mathrm{~km}$, pasando por $162 \mathrm{muni}-$ cipios (de un total de 262 municipios en el país) en trece de los catorce departamentos, donde reside más del 70 por ciento de la población. Provee agua para uso doméstico, agropecuario, pesquero e industrial a gran parte del país y genera casi 50 por ciento de la energía a nivel nacional.

Según las mismas empresas mineras transnacionales, los depósitos más importantes de oro y plata en El Salvador se encuentran en la zona norte, formando parte de un cinturón mesoamericano que se extiende desde Panamá hasta México. La mayoría de estos depósitos, en el caso de El Salvador, se ubican en la cuenca del río Lempa

Durante las administraciones de Calderón Sol (1994-1999) y de Francisco Flores (1999-2004), se aprobaron 29 permisos de exploración de minerales metálicos en esta zona, lo cual generó una amenaza grave al sistema hídrico de El Salvador, y al medio ambiente en general, y puso en grave peligro el desarrollo sostenible del país.

\section{Un balance desfavorable entre costos y beneficios de la minería metálica}

Hay una realidad curiosa en los países en vías de desarrollo alrededor del mundo donde explotan metales preciosos, como oro y plata. Con frecuencia, en estos países, se encuentran minas ricas en minerales a la par de comunidades que viven en la absoluta pobreza. Ante este panorama cabe preguntarse ¿Cómo es posible que una comunidad que vive a la par de una mina de oro viva en la absoluta pobreza? ¿Quiénes se benefician realmente de la minería de oro y quienes pagan los costos?

Las empresas mineras argumentan que la minería genera empleo y un "boom económico" para comunidades a nivel local. No obstante, según estadísticas de la Organización Internacional del Trabajo (OIT) de las Naciones Unidas, en el 2010 el sector minero generaba 3,7 millones de empleos a nivel mundial de un total de 5 mil millones, es decir el 0,074 por ciento (Fraser Institute, s. f.). Además, el empleo es principalmente para personas extranjeras, ya tecnificadas y experimentadas en la industria minera, no para la gente de las comunidades cercanas. Al mismo tiempo, la minería 
de oro está altamente mecanizada, por lo que requiere poca mano de obra y los empleos ofrecidos tienden a ser los más peligrosos, los más inestables y los peor pagados.

Otro elemento importante a considerar es que las minas de hoy tienden a tener vidas muy cortas. En el caso de la mina Marlin en San Marcos, Guatemala, se planteó una vida de explotación de solo 10 años, por lo que ya está en proceso de cierre. La mina San Martin en Valle de Siria, Honduras, cerró después de 9 años y a la mina El Dorado en Cabañas, El Salvador, se le pronostica una vida de solo 6 años, aproximadamente.

Cualquier empleo que genera la minería de oro en Centroamérica será de poca duración, tiempo suficiente para destruir los bosques y contaminar el suelo y el agua, poniendo en peligro la agricultura, la pesca, la ganadería y las demás formas tradicionales de vida en las comunidades cercanas. Esto es el "boom económico" que se hereda de la minería de oro. Además, los costos de la destrucción ambiental y la amenaza a la viabilidad de las comunidades más cercanas los pagan los pobres, mientras que las ganancias y otros beneficios de la minería se quedan con los dueños de las empresas.

Al respecto, Thomas M. Power (2008: 6), en su estudio sobre la minería en Centroamérica, plantea que:

En general, el desarrollo minero no ha traído prosperidad sustancial a los trabajadores, comunidades o países. Con algunas excepciones, países especializados en la explotación de minerales han experimentado tasas significativamente más lentas de crecimiento económico que otros durante el último cuarto de siglo.

La abundancia de evidencia sobre la relación entre la minería metálica y la pobreza en países en vías de desarrollo ha llevado a muchos analistas a hablar en términos de la "maldición de los recursos naturales" en lugar de los beneficios (Oxfam, 2009). La razón de fondo de esta relación sorprendente es que los costos económicos, sociales y ambientales a largo plazo para los países en vías de desarrollo son mucho más grandes que los beneficios, que tienden a ser pocos y de muy corto plazo.

\section{El derecho de la ciudadanía a saber y decidir sobre la minería metálica}

Una consideración final, y tal vez la más importante, que se debe tomar en cuenta en el debate sobre la minería metálica en El Salvador, es el derecho de la ciudadanía, especialmente de las comunidades más afectadas, de saber y decidir sobre esta industria; es decir, de manejar información independiente y verídica y expresar su voz y voluntad, de forma vinculante, en la toma de decisiones.

Desde hace décadas, a nivel mundial las empresas mineras han argumentado que la minería metálica es pilar esencial para el crecimiento económico y para el desarrollo de las sociedades de nuestro planeta por lo que sería imposible, irresponsable e 
ilógico sostener una posición de prohibición de la minería en cualquier parte del mundo. No obstante, comunidades circunvecinas a proyectos mineros en Centroamérica, y alrededor del mundo, quienes más sufren en carne propia el impacto de la minería metálica, están levantando su voz con más fuerza en protesta por los pocos beneficios y los grandes costos económicos, sociales y ambientales que trae esta industria.

En el caso de El Salvador, desde hace más de diez años, comunidades afectadas, junto con organizaciones de la sociedad civil, ONG, ambientalistas, la Conferencia Episcopal de la Iglesia católica, universidades como la Universidad Centroamericana "José Simeón Cañas" (UCA), la Produraduría para la Defensa de los Derechos Humanos, exfuncionarios de gobierno, personalidades respetadas y gupos aliados en el exterior reclaman por la aprobación de políticas estatales que prohiban la minería metálica en este país.

En este contexto, para dar voz a las comunidades cercanas a los depósitos minerales en El Salvador, el Instituto Universitario de Opinión Pública (IUDOP) de la UCA, en el año 2007, presentó un sondeo de opinión pública llevado acabo en 23 de los municipios más amenzadas con proyectos mineros (IUDOP, 2008). El estudio mostró que 67,6 por ciento de la población encuestada consideraba que los proyectos mineros contribuirían poco o nada al desarrollo económico de El Salvador y que 62,5 por ciento consideraba que El Salvador no era un país apropiado para esta industria.

En junio del año 2015, la UCA presentó un segundo sondeo (IUDOP, 2015) en el cual mostraba un incremento importante en el nivel de rechazo entre la ciudadanía hacia esta industria. En la nueva encuesta, el 76 por ciento de la población se mostró inconforme con la apertura de proyectos mineros en su municipio; el 79,5 por ciento expresó que El Salvador no era un país apropiado para la minería metálica y 77 por ciento consideró que el Estado salvadoreño debe prohibir definitivamente la minería metálica en el país.

A pesar de la crítica situación de desempleo en las comunidades encuestadas, el 86,5 por ciento de la población indicó que no tendría interés en trabajar en una mina, al tiempo que el 89,9 por ciento opinó que la contaminación del agua a consecuencia de la minería metálica sería muy o algo grave y aproximadamente ocho de cada diez personas consideraron que, por estos niveles de contaminación, las formas tradicionales de vida en el país, como la agricultura, ganadería y la pesca, serían algo o muy dañadas.

El 67,6 por ciento se opuso al desarrollo de proyectos de minería metálica en países fronterizos, por el impacto que podría tener en las fuentes compartidas de agua, y 92,8 por ciento de las personas que sabían de la actual demanda de Pacific Rim y su nuevo dueño, OceanaGold, contra el Estado salvadoreño afirmó que está en contra de que una empresa extranjera obligue al país a dar permisos para la explotación minera. 


\section{Conclusiones finales: políticas públicas y la buena gobernanza}

Las páginas anteriores detallan una situación de vulnerabilidad, amenaza, riesgo y resistencia frente a la ofensiva feroz de empresas mineras transnacionales en El Salvador. Además, vislumbran el enorme reto que existe hoy en día para países en vías de desarrollo que tratan de generar políticas públicas, programas y prácticas en defensa del medio ambiente, la vida humana, los derechos a la ciudanía y la soberanía nacional frente los intereses corporativos de las empresas transnacionales. Más de fondo, plantean la urgencia de definir nuevas paradigmas para el desarrollo en estos países con alternativas al modelo extractivista actual.

El desarrollo económico siempre requiere de la toma de decisiones sobre la forma de gestionar recursos naturales finitos y limitados para convertirlos en bienes que mejoran la vida de las personas. Sin embargo, durante el último siglo, el modelo de desarrollo a través de la extracción y exportación de materias primas ha devastado los recursos naturales de tal manera que pone en riesgo el desarrollo, a largo plazo, a cambio de ganancias y beneficios inmediatos.

La búsqueda de nuevos paradigmas más sostenibles empieza con la formulación y ejecución de políticas, programas y prácticas que incorporan principios de sostenibilidad, equidad, participación, prevención, eficiencia y transparencia. Hay dos conceptos clave que acuerpan estos principios y que deben tomarse en cuenta frente a la amenaza de la minería metálica al agua en nuestro mundo con sed:

\section{La Gestión Integrada de Recursos Hídricos (GIRH)}

El concepto de la gestión integrada de los recursos hídricos surgió a principios del siglo XXI como respuesta ante el creciente deterioro del medio ambiente en el planeta, especialmente del agua; y hoy en día, se ha afianzado en Estados Unidos, Europa, Australia y, cada vez más, en países en vías de desarrollo alrededor del mundo.

Este concepto no plantea una ruta escrita en piedra para el manejo del agua. Más bien, reconoce que cada contexto requiere de respuestas distintas. Promueve, como fórmula esencial para el desarrollo sostenible, la premisa de que el manejo eficiente del agua depende del uso eficiente de la tierra en el marco de un enfoque integral de cuenca.

Además, reconoce que son los pobres de este mundo quienes sufren más con el mal manejo del agua y promueve principios de equidad social con eficiencia económica y sostenibilidad ambiental. Al tiempo que enfatiza en la participación amplia de actores claves (stakeholders) en la toma de decisiones sobre el manejo del agua, al plantear la importancia de la transparencia y cooperación entre usuarios. A la vez, lucha por generar entornos normativos propicios para el logro de resultados consensuados a través de procesos dinámicos de toma de decisión. 
De este modo se le considera un concepto político porque se trata del poder, priorizando el poder de las comunidades más afectadas de decidir sobre el acceso, el uso y el cuido de sus fuentes de agua dulce.

\section{El Consentimiento previo, libre e informado (CPLI)}

El concepto de consentimiento previo, libre e informado (CPLI) surge en el Convenio 169 de la Organización Internacional del Trabajo (OIT, 1989) y se reconfirma en la Declaración de los Derechos de los Pueblos Indígenas de las Naciones Unidas (ONU, 2007). Se establece como principio de participación ciudadana en la toma de decisiones sobre proyectos económicos que impactan la vida de los pueblos indígenas y se ha vuelto norma de buena gobernanza y un derecho para todas las comunidades afectadas por actividades extractivas.

En el contexto del CPLI, consentimiento se refiere al derecho de las comunidades a la consulta de buena fe con participación plena y equitativa en la toma de decisiones. Mientras que Previo implica consultar antes de autorizar cualquier proyecto extractivo, libre significa la ausencia de coerción, intimidación o manipulación y el respeto a las formas y mecanismos tradicionales de consulta de las comunidades afectadas; e informado consiste en proporcionar suficiente información de forma objetiva y verídica sobre los costos y beneficios de cualquier actividad extractiva.

En el caso de El Salvador, la aplicación de los conceptos de GIRH y de CPLI, en el marco de la política gubernamental relacionada específicamente con la protección de los recursos hídricos y la minería metálica, se promueven a través de 3 grandes luchas estratégicas que la ciudadanía organizada viene impulsando por más de una década:

\section{La lucha por el reconocimiento del agua como derecho humano}

La lucha por el reconocimiento del agua como derecho humano se enfoca en la reforma del artículo 69 de la Constitución de la República. Ha sido apoyado por las Naciones Unidas y las coaliciones de la sociedad civil, con la oposición de partidos políticos de derecha y la gran empresa, que ven al agua como mercancía, sujeto a la privatización.

\section{La lucha por la Ley General de Aguas}

La Ley General de Aguas promovida por la sociedad civil organizada y el gobierno actual de El Salvador establece los principios, los valores, la institucionalidad y los procedimientos para un manejo eficiente, equitativo y sostenible del recurso hídrico en ese país. Además, prohíbe la privatización de este recurso tan vital para la vida, 
promueve la participación ciudadadana a nivel local y nacional, plantea un ente rector público para su gestión y enfatiza la gestión integral del agua con enfoque de cuenca.

\section{La lucha por la Ley de Prohibición de la Minería Metálica}

La lucha para la prohibición de la minería metálica promueve una legislación que cierre la puerta a la minería metálica, en todas sus formas, y al uso de químicos tóxicos, como cianuro y mercurio. A la vez, propone regular de mejor manera la minería no metálica en el país. Para contribuir a este esfuerzo, la Universidad Jose Simeón Cañas (UCA) ha elaborado una propuesta de ley que plentea el siguiente objetivo:

\section{Art. 1.-}

La presente ley tiene por objeto prohibir cualquier actividad de exploración, explotación, procesamiento y otras relacionadas con yacimientos de minerales metálicos en cualquiera de las formas posibles. También prohíbe el uso de sustancias tóxicas para la salud y los ecosistemas, tales como el cianuro y mercurio, entre otros.

Asimismo la ley tiene por objeto normar el ejercicio de los derechos soberanos del Estado salvadoreño, para administrar, regular y controlar el sector minero no metálico, de conformidad con los principios de sostenibilidad, precaución, prevención y eficiencia. Esto incluye la regulación de los aspectos relacionados con la prospección, exploración, explotación, procesamiento, almacenamiento, transporte y comercialización de los recursos minerales no metálicos existentes en el suelo y subsuelo del territorio de la República. Se exceptúan de lo anterior los hidrocarburos en estado líquido o gaseoso, que se regulan en leyes especiales y la extracción de sal obtenida por procesos de evaporación de aguas marinas.

La mayoría de activistas y analistas en El Salvador no plantea la prohibición de la minería metálica en todo el mundo. Se reconoce la importancia de minerales para el desarrollo de los países centroamericanos, pero no en cualquier lugar del planeta, ni con cualquier tecnología. Dónde se hace la minería, cómo se lleva a cabo dicha actividad y a quién se benefícia, son consideraciones clave para cualquiera comunidad y país con recursos minerales.

En países con ecosistemas vulnerables y sin licencia social (el consentimiento de la ciudadanía), como es el caso de El Salvador, la opción de prohibición es la más lógica y debe ser aceptada por las empresas transnacionales, sin amenazas de demandas y sin el atropello de la soberanía nacional. Ante esto, se deben priorizar los derechos comunitarios sobre los intereses corporativos transnacionales, y los países en vías de desarrollo deben buscar modelos más justos, más humanos y más sostenibles para asegurar el "Buen Vivir" de los pueblos y un camino más seguro hacia el desarrollo. 


\section{Bibliografía}

Broad, Robin y Cavanagh, John. Like Water for Gold in El Salvador. The Nation, 2011. Web. <http://www.stumbleupon.com/su/32m7Rr/www.thenation.com/article/162009/ water-gold-el-salvador $>$.

"Cedió un Dique con Aguas Contaminadas de una Mina y hay 17 Muertos". La Gaceta [Tucumán] 6 de nov. 2015: Mundo. N.pág. Web. <http://www.lagaceta.com.ar/nota/660092/ mundo/brasil-cedio-dique-aguas-contaminadas-mina-hay-17-muertos.html>.

“China a la conquista de Latinoamérica”. Sepa más. RT, 2015.Web. <https://actualidad.rt.com/ actualidad/192245-china-inversiones-proyectos-america-latina $>$.

Comisión Pastoral Paz y Ecología (COPAE) de la Diócesis de San Marcos, "Los Problemas Creados por la Mina Marlin - Goldcorp - en San Marcos, Guatemala", (s. f.).Web. $<$ http://copaeguatemala.org/articulosCOPAE/Los\%20Problemas $\% 20$ creados $\% 20$ por $\% 201 \mathrm{l} \% 20 \mathrm{mina} \% 20 \mathrm{Marlin}$. html $>$.

“Contaminación de las Aguas del Río Lara por Derrame de Cianuro". Tribunal Centroamericano del Agua, Resumen Ejecutivo de Casos Segunda Audiencia de Juzgamiento 15-19 marzo, (2004) HONDURAS. Web. <http://tragua.com/wpcontent/uploads/2012/04/ caso_rio_lara.pdf $>$.

“¿Cuántos trabajos dependen de la industria minera?”. Realidad Minera Fraser Institute. Web. $<$ http://www.miningfacts.org/Economy/How-many-jobs-depend-on-the-miningindustry/?LangType $=1034>$.

"Cuidemos la Casa de Todos". La Conferencia Episcopal de la Iglesia católica, Caritas El Salvador, 2007. Web. <http://www.caritaselsalvador.org.sv/documentos.html >.

“C169 Convenio sobre pueblos indígenas y tribales". Organización Internacional de Trabajo (OIT), 1989. Web.<http://www.ilo.org/wcmsp5/groups/public/@ed_norm/@normes/ documents/publication/wcms_100910.pdf>.

“Declaración de los Derechos de los Pueblos Indígenas de las Naciones Unidas”. Organización de las Naciones Unidas (ONU), 13 de sept. de 2007. Web. <http://www.un.org/esa/ socdev/unpfii/documents/DRIPS_es.pdf>.

"Desarrollo sostenible". Asamblea General de las Naciones Unidas. Organización de las Naciones Unidas [ONU]. Web. <http://www.un.org/es/ga/president/65/issues/sustdev.shtml >.

Instituto Universitario de Opinión Pública [Iudop] "Conocimientos y percepciones hacia la minería en zonas afectadas por la incursión minera". Instituto Universitario de Opinión Pública. Universidad Centroamericana José Simeón Cañas. Resumen ejecutivo, 2015 Web. $<$ http://www.uca.edu.sv/publica/iudop/Web/2008/Resumen-IUDOP-Mineria.pdf>.

Instituto Universitario de Opinión Pública [Iudop] “Opiniones y percepciones hacia la minería metálica en El Salvador". Boletín de prensa. Año XXIX, N². Instituto Universitario de Opinión Pública. Universidad Centroamericana José Simeón Cañas. Resumen ejecutivo, 2015 Web. <http://www.uca.edu.sv/iudop/wp-content/uploads/Bolet\%C3\%ADn-deprensa.pdf $>$.

Maroquin, David, "CIADI Retrasa Fallo Caso Pacific Rim”, El Salvador.com [El Salvador], 31 de Agosto, 2016: Sucesos. Web. Septiembre, 2016. <http://www.elsalvador.com/articulo/ sucesos/fallo-del-ciadi-entrepacific-rim-salvador-conocera-hasta-mediados-septiembre-123754>.

Mickinley, Andrés. El Derecho de saber y decidir sobre la minería metálica. El caso de El Salvador. San Salvador: Talleres Gráficos, 2015 Iudop U Centroamericana José Simeón Cañas (UCA). 
Impreso-Web. <http://www.uca.edu.sv/iudop/wp-content/uploads/EL-DERECHODE-SABER-Y-DECIR-.pdf $>$.

Mckinley, Andrés. La amenaza de la minería metálica en un mundo con sed. San Salvador: UCA Editores, 2015 Iudop U Centroamericana José Simeón Cañas (UCA). Impreso-Web. <http:// www.uca.edu.sv/iudop/wp-content/uploads/La-Amenaza-de-la-Mineri\%C3\%ACaMeta $\%$ C3\%AClica-en-un-Mundo-con-Sed.pdf $>$.

Mckinley, Andrés. Mitos y realidades de la minería de oro en Centroamérica. N.P. Cáritas El Salvador., s. f. Web. <http://www.caritaselsalvador.org.sv/docs/mitosyrealidades.pdf $>$.

Mendoza, B. y Sosa, B. “90 \% de aguas superficiales del país están contaminadas" La Prensa Gráfica. [El Salvador], 2016. Web. http://www.laprensagrafica.com/2016/03/21/90-deaguas-superficiales-del-pais-estan-contaminadas $>$.

“Microscópico Recuperación de Oro" Bueno Saber, s. f. Web. <http://bueno-saber.com/aficiones-juegos-y-juguetes/ciencia-y-naturaleza/microscopico-recuperacion-de-oro.php>.

Movimiento Mesoamericano Contra el Modelo Extractivo Minero, "Mineria Contamina con Cianuro Rio en Nicaragua"; Estrategias por la Defensa de la Tierra y el Territorio. Web. <http://movimientom4.org/2013/05/minera-contamina-con-cianuro-rio-ennicaragua-denuncia-diputado/>.

Morales, Iván "El Salvador: El desarrollo pasará por reducir el riesgo de desastres". OXFAM International, 2014. Web. <https://blogs.oxfam.org/es/blogs/14-02-20-el-salvador-eldesarrollo-pasara-por-reducir-el-riesgo-de-desastres $>$.

Organización de las Naciones Unidas para la Agricultura y la Alimentación [FAO], "Memoria Seminario Regional sobre los Sistemas dentro Energéticos Optimizados para el Desarrollo Rural y la Protección Ambiental", Depósitos de documentos de la FAO, 1993. Web. <http:// www.fao.org/docrep/006/AD097S/AD097S05.htm>.

Ortúzar, F. y Rodríguez, K. “¡Cuidado!: La minería propone llevar sus desechos al mar”. Asociación Interamericana para la Defensa del Ambiente [AIDA], 2015. Web. <http:// www.aida-americas.org/es/blog/cuidado-la-mineria-propone-llevar-sus-desechosal-mar>.

Oxfam, "Contra la Maldición de los Recursos Naturales", 2009. Web. <https://www.oxfam. org/sites/www.oxfam.org/files/file_attachments/bp134-lifting-the-resource-cursesummary-sp_3.pdf $>$.

Power, Thomas. Minería de metales y desarrollo sostenible en Centroamérica. Una valoración de costos y beneficios. Oxfam América, 2008.

Rivas, German. "El Salvador con el peor escenario hídrico en C.A". La Prensa Gráfica. [El Salvador] 23 de nov. 2010: Social N. pág. Web. <http://www.laprensagrafica.com/elsalvador/lodeldia/153343-el-salvador-con-el-peor-escenario-hidrico-en-ca>.

Ross, Michael. Sectores Extractivas y Pobreza. Ciudad de California: U de California, 2001.

Sachs, Jeffrey y Warner, Andrew M. La Abundancia de Recursos Naturales y el Crecimiento Económico. Massachusetts: Cambridge U de Harvard, 1997.

Saenz, Rebeca. "Resumen Ambiental Nacional El Salvador". Programa de Naciones Unidas para el Medio Ambiente (PNUMA), 2010. Web. <http://www.pnuma.org/publicaciones/NES\%20El\%20Salvador\%20FINAL-\%2016-12-10\%20-\%20edited.pdf>.

Shahid, A. y Qiren, L. "Tailings Dam Failures: a Review of the Last Hundred Years". Waste Geo technics, 2010. Web. <http://www.infomine.com/library/publications/docs/ Azam2010.pdf>. 
Sumi, L. y Gestring, L. "Polluting The Future: How mining companies are contaminating our nation's waters in perpetuity" Earthworks, 2013. Web. <https://www.earthworksaction.org/files/publications/PollutingTheFuture-FINAL.pdf $>$.

Tesoro o Problema: La Minería Metálica en Países en Vías de Desarrollo. Departamento de Minería: Grupo Banco Mundial, 2002.

Andrés McKinley. Estadounidense, cuenta con una maestría en Administración de Salud y ha trabajado durante 46 años con organizaciones internacionales de desarrollo. En los últimos 15 años se ha especializado en el tema de las industrias extractivas (minería). Su trabajo le ha permitido conocer de cerca tanto los beneficios como los costos económicos, sociales, culturales y ambientales de la minería metálica en Mesoamérica (México, Guatemala, El Salvador, Honduras, Nicaragua, Costa Rica, Panamá), Suramérica (Colombia, Ecuador, Perú, Bolivia), África (Mali, Senegal, Liberia, Ghana) y Estados Unidos de Norteamérica. Reside en Centroamérica desde 1977. Contacto: amckinley45@gmail.com 